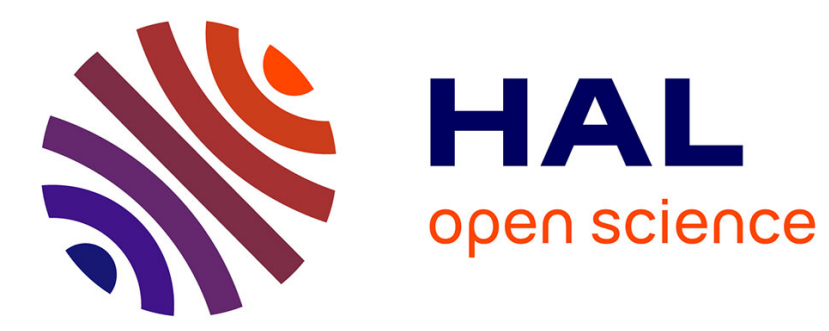

\title{
Subnanowatt Opto-Molecular Generation of Localized Defects in Chiral Liquid Crystals
}

Charles Loussert, Supitchaya Iamsaard, Nathalie Katsonis, Etienne Brasselet

\section{To cite this version:}

Charles Loussert, Supitchaya Iamsaard, Nathalie Katsonis, Etienne Brasselet. Subnanowatt OptoMolecular Generation of Localized Defects in Chiral Liquid Crystals. Advanced Materials, 2014, 26 (25), pp.4242-4246. 10.1002/adma.201400811 . hal-01063668

\section{HAL Id: hal-01063668 https://hal.science/hal-01063668}

Submitted on 26 Feb 2018

HAL is a multi-disciplinary open access archive for the deposit and dissemination of scientific research documents, whether they are published or not. The documents may come from teaching and research institutions in France or abroad, or from public or private research centers.
L'archive ouverte pluridisciplinaire HAL, est destinée au dépôt et à la diffusion de documents scientifiques de niveau recherche, publiés ou non, émanant des établissements d'enseignement et de recherche français ou étrangers, des laboratoires publics ou privés.

\section{다)(1) $(5$}

Distributed under a Creative Commons Attribution - NonCommerciall 4.0 International 


\title{
Subnanowatt Opto-Molecular Generation of Localized Defects in Chiral Liquid Crystals
}

\author{
Charles Loussert, Supitchaya lamsaard, Nathalie Katsonis, * and Etienne Brasselet*
}

Liquid crystalline materials are well-known to exhibit various kinds of structural defects, whose space-variant optical properties are actually useful for a number of applications, including recently developed integrated optical vortex generators. ${ }^{[1-5]}$ Since topological defects cannot be transformed one to another by mere continuous changes of the material texture, these structures are highly relevant for information storage. An illustrative example is provided by frustrated chiral liquid crystals films, in which different localized metastable defect structures can be written by either structured ${ }^{[6]}$ or unstructured ${ }^{[7]}$ light beams. Such developments can be viewed as spatially controlled versions of randomly distributed defect structures generated by uniform quasistatic electric fields as shown in Refs. ${ }^{[8,9]}$ four decades ago, see also Ref. ${ }^{[10,11]}$ for more recent works.

Optically induced metastable defect structures in chiral liquid crystals have been studied regarding their capabilities in terms of reconfiguration, ${ }^{[6]}$ large-scale integration, ${ }^{[12]}$ threedimensional photonic architectures, ${ }^{[13]}$ phase masks up to millimeter square area ${ }^{[14]}$ or arrays of microscopic optical vortex generators. ${ }^{[15]}$ To date their application potential however remains limited. Indeed, the reversible generation of a localized defect structure by focused light beams typically requires a 10-100 $\mathrm{mW}$ power budget with typical intensities ranging from $1-10 \mathrm{~kW} \mathrm{~cm}^{-2}$ for low numerical aperture focusing elements up to $0.1-1 \mathrm{MW} \mathrm{cm}^{-2}$ for high numerical aperture. ${ }^{[6,12]}$ This implies a "point-by-point" laser writing strategy and, consequently, crippling fabrication times when considering the realization of large and dense arrays of them. ${ }^{[12,15]}$ Still, a few years ago, an approach relying on photo-active achiral dopants has been proposed to reduce the power budget by a few orders of magnitude, down to $10 \mu \mathrm{W} \cdot{ }^{[16]}$ However, such an improvement came at the expense of reversibility, as intrinsically irreversible photo-chemical processes are involved.[16] Combining optical sensitivity to reversibility would thus constitute a significant improvement for applications where addressability is a key issue.

\footnotetext{
C. Loussert, E. Brasselet

Université de Bordeaux, CNRS

Laboratoire Ondes et Matière d'Aquitaine

F-33400, Talence, France

E-mail: e.brasselet@loma.u-bordeaux.fr

S. lamsaard, N. Katsonis

Laboratory for Biomolecular Nanotechnology

MESA+ Institute for Nanotechnology

University of Twente

POBox207,7500AE , Enschede, TheNetherlands

E-mail: n.h.katsonis@utwente.nl
}

Here we report on the reversible topological structuring of frustrated chiral liquid crystals films by light. In so doing, we also demonstrate the formation of various kinds of chiral defect structures at the micron-scale and subnanowatt optical power level. The demonstration is made by harnessing the chirality locally and at the molecular scale. Noticeably, our results unlock inherent limitations associated to the molecular photo-activation of textures using spatially extended illumination, see Ref. ${ }^{[17]}$ for thin films with perpendicular orientational boundary conditions and Ref. ${ }^{[18]}$ for parallel alignment, which prevents from local control of the liquid crystal topology. Our molecular approach basically relies on the use of chiroptical molecular switches, ${ }^{[19,20]}$ which allows the use of ultra-low irradiation powers and intensities compared to the state of the art based on passive liquid crystals while preserving the reversibility of the process. The use of light-responsive switches as molecular dopants for chiral liquid crystals has been reported before, and used successfully in controlling the pitch, handedness and orientation of the cholesteric helix, ${ }^{[21]}$ to convert light into macroscopic motion, ${ }^{[22,23]}$ or to develop photo-switchable surface topologies. ${ }^{[24]}$ However, to the best of our knowledge, they have not been used to control the structure of topological defects so far.

Experiments are made by using thin films of cholesteric liquid crystals. The chiral nature of a cholesteric is characterized by its helical supramolecular ordering having a pitch $p$, where $p$ is the distance over which the director $\mathbf{n}$ (a unit vector that defines the local average molecular orientation of the liquid crystal, with $\mathbf{n}$ being equivalent to $-\mathbf{n}$ ) rotates by $2 \pi$ [Figure 1(a)]. In particular, when the twisted elastic distortions associated with the cholesteric helix are incompatible with the orientational boundary conditions, the molecular chirality is not expressed at the supramolecular level and the director field at rest is consequently unwound. ${ }^{[25]}$ Such a "geometric frustration" can occur when the boundary conditions on both sides of the film promote a perpendicular (homeotropic) orientation of the director. More precisely, for a film of thickness $d$ submitted to homeotropic boundary conditions, the cholesteric helix is unwound when the pitch $p$ is larger than a critical pitch value $p_{c}$ [Figure 1(b)], but forms textures when $p / p_{c}{ }^{[10]}$ [Figure 1(c)]. In practice, the unwinding/winding transition occurs for the critical thickness-to-pitch ratio $d / p_{c}=K_{3} /\left(2 K_{2}\right),{ }^{[26]}$ where $K_{2}$ and $K_{3}$ are the twist and bend Frank elastic constants of the liquid crystal. Based on this, our proposition is that if, in a frustrated geometry $\left(p>p_{c}\right)$, a sufficient pitch decrease down to $p<p_{c}$ could be promoted by a spatially controlled external trigger, this would lead to a remote-control structural winding transition and subsequent topological structuring.

To test this idea, we designed a cholesteric liquid crystal that reversibly winds under optical irradiation, by using light as 
(a)

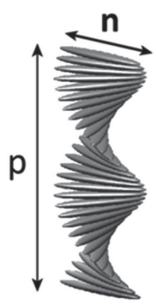

(b)

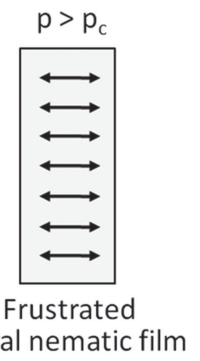

(c)

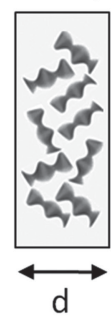

(d)
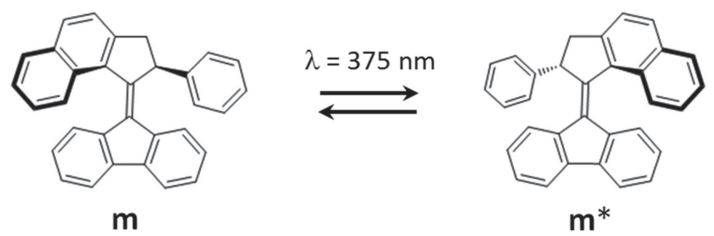

(f)

(e)

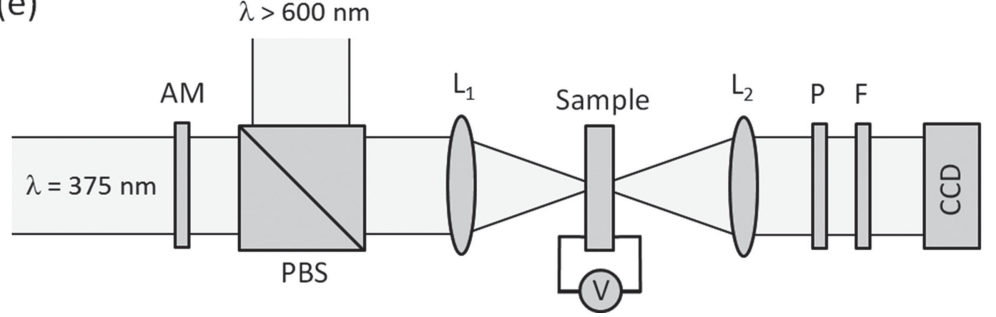

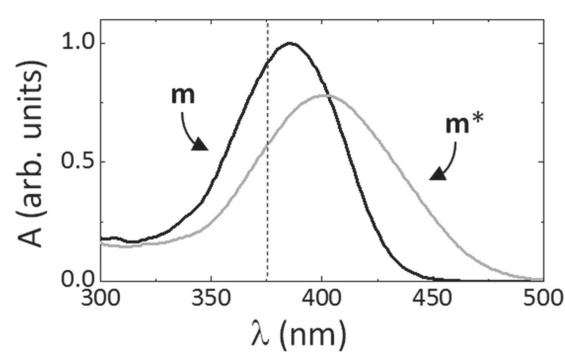

Figure 1. a) Representation of the helical ordering of a cholesteric liquid crystal, with $p$ the helical pitch and $\mathbf{n}$ the director. b) Confinement of a cholesteric liquid crystal in a cell of thickness $d$ that imposes a perpendicular molecular alignment at the film boundaries, with $p<p_{c}$, which leads to the frustration of the helical molecular ordering. By contrast, twisted defect texture takes place when $d / p$ is large enough, as shown in panel (c). In our system, the cholesteric pitch $p$ is optically adjusted at fixed $d$ under irradiation at $\lambda=375 \mathrm{~nm}$ owing to the use of a photo-responsive chiral molecular dopant $\mathbf{m}$. The photo-chemical behavior of $\mathbf{m}$ is illustrated in panel $\mathbf{d}$ ) where $\mathbf{m} *$ is the photo-activated isomer of $\mathbf{m}$. The corresponding inversion of the molecular chirality is used to trigger the unwinding/winding transition at the supramolecular level. e) Experimental setup, see text for details. AM: amplitude mask; PBS: polarizing beam splitter; $\mathrm{L}_{1,2}$ : lenses; $\mathrm{V}$ : low-frequency $(1 \mathrm{kHz})$ applied voltage; P: polarizer; F: filter for UV light; CCD: video imaging device. $\mathrm{f}$ ) Room temperature UV-visible absorbance spectrum $A(\lambda)$ of the molecular switch at $10^{-4} \mathrm{M}$ in hexane. The black curve refers to the spectrum of $\mathbf{m}$ whereas the gray curve corresponds to the spectrum of $\mathbf{m} *$.

an external control parameter for the molecular chirality. For this purpose, we introduce a photo-switchable chiral molecule into an (achiral) nematic liquid crystal. Here we work at room temperature by using E7 mixture (from Merck) as the nematic host, which is doped with a homemade molecular switch, $\mathbf{m}$ [Figure 1(d)], which is known to behave as a light-driven molecular motor that undergoes unidirectional rotation under irradiation with ultraviolet (UV) light. ${ }^{[27]}$ In practice, under irradiation at $\lambda=375 \mathrm{~nm}$, structural changes associated with the photoisomerization $\mathrm{m} \rightarrow \mathrm{m}^{*}$ express at the supramolecular scale as large cholesteric pitch variations ${ }^{28,29]}$ (see absorption spectra of both forms in Figure 1(f)). In its stable form, $\mathbf{m}$ induces a right-handed cholesteric helix in E7 and, since we aim at optically reducing the pitch starting from an initial situation with $p>p_{c}$, a shape-persistent chiral molecular co-dopant inducing a left-handed twist is also added to the cholesteric mixture in order to overcompensate for the right-handed twist promoted by $\mathbf{m}$. For this purpose, we chose the shape-persistent chiral dopant (R)-2,2'-(1,3-propylenedioxy)-1,1'-binaphthalene. By using concentrations of $0.8 \mathrm{wt} . \%$ and $1.0 \mathrm{wt} . \%$ for the photoresponsive and shape-persistent chiral dopants respectively, our co-doping procedure yields a photo-responsive left-handed cholesteric helix that winds upon UV irradiation from the initial natural pitch $\mathrm{p}=18 \mu \mathrm{m}$ to $\mathrm{p}=1.7 \mu \mathrm{m}$ once photo-equilibrium is reached, which we evaluated from the Granjean-Cano wedge method. ${ }^{[30]}$ Since the elastic constants of E7 are $K_{2}=7.4 \mathrm{pN}$ and $K_{3}=16.5 \mathrm{pN}$ at $25^{\circ} \mathrm{C}$, we get $d / p_{c} \sim 1.1$ and we thus opt for a homeotropic cell of thickness $d=10 \mu \mathrm{m}$. As expected, the cholesteric helix is unwound at rest whereas frustration can be relieved under irradiation with UV light.
Our setup is shown in Figure 1(e). The writing light field is provided by a diode-pumped solid-state laser operating at $\lambda=375 \mathrm{~nm}$ in the fundamental Gaussian mode and is combined to a polychromatic imaging illumination light from an halogen lamp by means of the polarizing beam splitter PBS. Importantly, the spectral range of the illumination field is restricted to $\lambda>600 \mathrm{~nm}$ in order to prevent its absorption by any of the compounds $\mathbf{m}$ or $\mathbf{m}^{*}$, see Figure $1(\mathrm{f})$. The lens $\mathrm{L}_{1}$ allows either (i) focusing the writing field on the sample placed at a distance $z$ from the focal spot whose diameter equals $d$ or (ii) imaging, in the plane of the sample, an amplitude mask AM placed in the rear of PBS. The lens $\mathrm{L}_{2}$ allows real-time monitoring of the light-induced topological structuring with a CCD camera in front of which are placed a polarizer $\mathrm{P}$ and a filter $\mathrm{F}$ that blocks the residual UV light. In practice, two optical winding regimes may take place depending on the ratio between the characteristic diameter $d$ of the illuminated area and the film thickness $d$. Firstly, when $D \sim d$, topological microstructuring is achieved and results in the formation of distinct topological structures. Secondly, when $D \gg d$, large scale textures are observed, which offers the possibility to create any spatially extended optical pattern, i.e., image photo-inscription, as reported in ${ }^{[17,18]}$ for different kinds of anchoring conditions. The present work mainly focuses on the former case, that constitutes the novelty of the study.

Considering the situation $D \sim d$, the transition to a wound state upon laser irradiation at subnanowatt power level is experimentally ascertained by the observation of a bright area on a black background under crossed-polarizers imaging. Different kinds of photo-stationary states are found depending on the 


\section{Molecular optical activation in active chiral films}
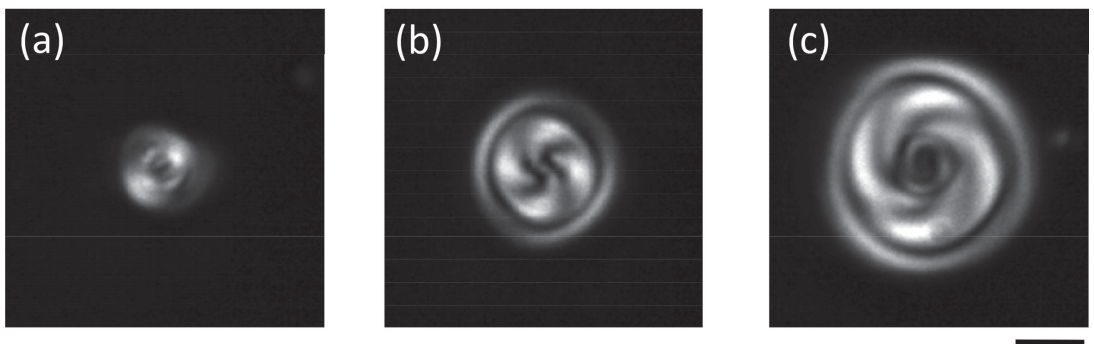

Supramolecular optical activation in passive chiral films
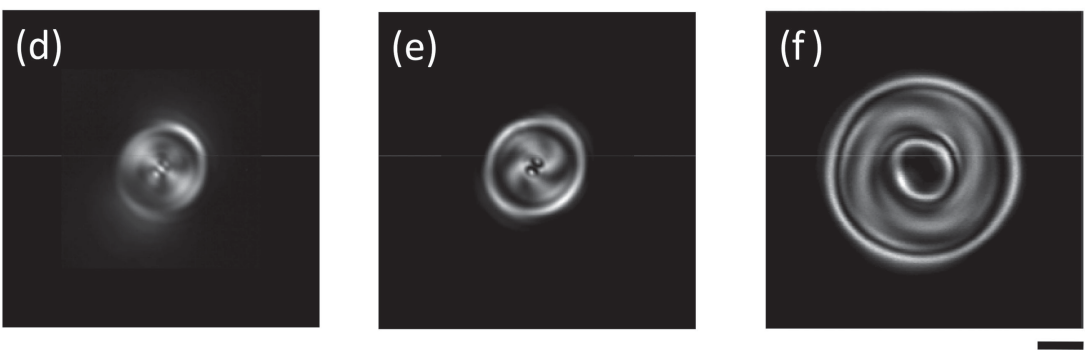

Supramolecular electrical activation in passive chiral films
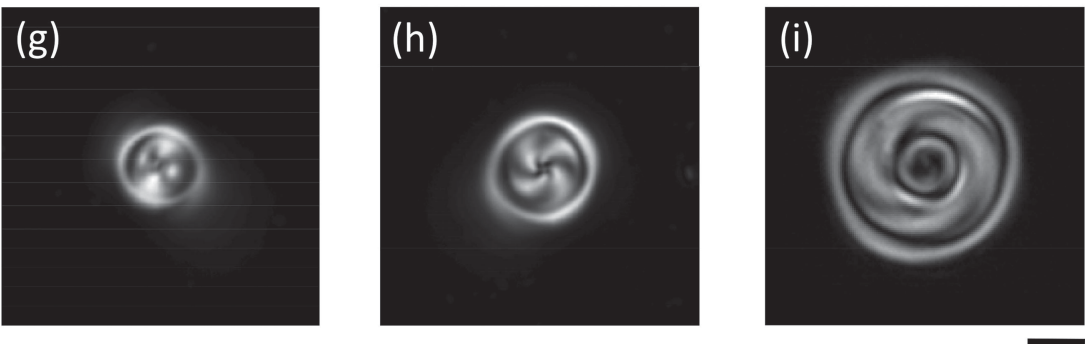

Figure 2. Topological structuring of a frustrated chiral nematic film. Upper row: local optomolecular triggering of defect structures in an active frustrated cholesteric film under Gaussian beam UV illumination. Experimental conditions: the beam waist diameter is $10 \mu \mathrm{m}$ and the sample is placed at a distance $z=0,100$ and $500 \mu \mathrm{m}$ from the focal plane in panel (a), (b) and (c) that respectively correspond to the photo-stationary state under total incident power $0.53 \mathrm{nW}, 0.65 \mathrm{nW}$ and $1.04 \mathrm{nW}$. Middle row: local supramolecular optical activation of three different metastable structures in a passive frustrated cholesteric film under Gaussian beam visible illumination, see ${ }^{[7]}$ for details. Bottom row: spontaneous supramolecular electrical generation of three different metastable structures in a passive frustrated cholesteric film. Scale bar: $10 \mu \mathrm{m}$. All images refer to crossed linear polarizers imaging.

distance $z$, as shown in Figures 2(a), 2(b) and 2(c) that correspond to $D \simeq d(z=0), D \simeq 1.1 d(z=100 \sim \mu \mathrm{m})$ and $D \simeq 2.6 d$ $(\mathrm{z}=500 \mu \mathrm{m})$, respectively. The visual inspection of these structures suggests to identify them as 'torons' defect structures labeled T3- $n(n=1,2,3)$ following the classification scheme proposed in Ref. ${ }^{[6]}$ in the case of passive frustrated cholesteric films. It should be noted that the structure shown Figure 2(c) might also be the manifestation of a Hopf fibration, ${ }^{[31]}$ which could be verified by a $3 \mathrm{D}$ reconstitution of the director field, but is out of scope for our present study.

The above mentioned conclusion that distinct topological states are actually observed however calls two comments. Indeed, one may rightly argue that: (i) in, ${ }^{[6]}$ the laser-induced defect structures correspond to metastable distorted orientational states whereas our system, being fully reversible, eventually relaxes to the frustrated state after the writing light field is turned off and (ii) the crossedpolarizers images shown in Figures 2(a), 2(b) and 2(c) quantitatively differ from those shown in. ${ }^{[6]}$

To address these two points, we refer to a recent work performed in a $10 \mu \mathrm{m}$-thick frustrated film prepared by doping the nematic liquid crystal mixture MLC-2048 with the chiral compound S811 at 0.86 wt.\% concentration, which gives a $10.4 \mu \mathrm{m}$-pitch passive cholesteric material. In that case, distinct metastable defect structures that do not disappear once the excitation beam is turned off are observed, as illustrated in Figures 2(d), 2(e) and 2(f), see ${ }^{[7]}$ for details. Importantly, this unambiguously matches with the observations performed in the photo-active cholesteric, thereby demonstrating that Figures 2(a), 2(b) and 2(c) actually refer to distinct topological microstructures. Still we note that the comparison of the crossed linear polarizers images of the structures we observe with the torons shown $\mathrm{in}^{[6]}$ reveals minor differences that are likely originating in different optical imaging conditions.

We also repeated the above mentioned experiment but using a uniform quasistatic electric field as a trigger to achieve director reorientation. This is made possible since the film is provided with transparent electrodes at both ends, which allows to relieve frustration by applying a $100 \mathrm{kHz}$ low-voltage electric field (a few Volts) owing to a negative dielectric anisotropy $\left(\epsilon_{a}=-3.4^{[32]}\right)$ that tends to reorient the director in the plane of the film. Similarly to the case of optical reorientation, we observe the spontaneous electrical generation of a large number of randomly distributed distinct metastable defect structures across the sample. The structures shown in Figures 2(g), 2(h) and 2(i) clearly match with the structures obtained using UV/visible irradiation of active/passive films, which constitutes an original result itself worth to mention.

Noticeably, these results support previous experimental demonstration $^{[7]}$ that helical beams do not constitute a pre-requisite to the formation of various kinds of chiral topological states, in contrast to state-of-the-art approach that relies on the use of high-order optical vortex beams in order to achieve topological diversity in passive media. ${ }^{[6]}$ Moreover, the fact that all structures have a typical size of the order of the pitch emphasizes that the observed topological diversity is inherent to the very nature of the chiral liquid crystal material and not of the used reorientation strategy, be it (i) local or spatially extended and (ii) acting at the molecular or supramolecular scale.

From a quantitative point of view, the opto-molecular approach developed here reduces both the required the intensity and power triggers by typically eight orders of magnitude 
(a)
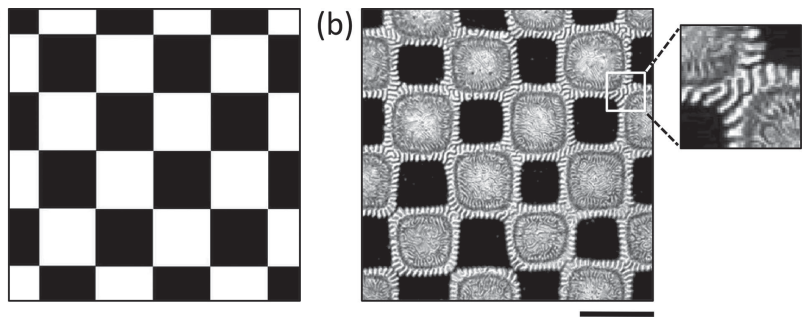

Figure 3. Steady-state checkerboard pattern image recorded under typ ical intensity of $1 \mathrm{~mW} \mathrm{~cm} \mathrm{~cm}^{-2}$. a) Original image of the amplitude mask. b) Imprinted pattern. The writing time of the order of $1 \mathrm{~min}$. Scale bar: $250 \mu \mathrm{m}$.

compared to the case of passive materials. A physical insight into such an optical gain is inferred from comparing the optical cross-sections involved in the reorientation processes for either the passive or the photo-responsive films. For passive films, the typical length scale over which the optical stimulus (i.e., the torque exerted by light) is effective corresponds to the liquid crystal coherence length ${ }^{[33]}$ and is of the order of the film thickness $d$. In contrast, for active films, it is the lightdriven reversal of the molecular chirality that activates a chiral elastic torque at the molecular level, hence associated with a typical length scale $a \sim 1 \mathrm{~nm}$. Comparing the corresponding cross-sections areas yields a ratio $(d / a)^{2} \sim 10^{8}$, namely an improvement by eight orders of magnitude.

We also report on the winding transitions that occur when the diameter of the writing beam is larger than the thickness of the photo-responsive film, i.e., $D \gg d$. Using spatially extended optical irradiation, the real-time monitoring of spatiallycontrolled cholesteric textures, which are reminiscent of the well-known fingerprint textures, becomes possible. Arbitrary patterns with square centimeter area can be obtained under milliwatt power illumination since intensity level of the order of $1 \mathrm{~mW} \mathrm{~cm} \mathrm{~cm}^{-2}$ is sufficient. The demonstration of large area photo-patterning merely requires the use of a stencil used as a binary amplitude phase mask that is imaged on the sample. With the present material, photo-stationary patterns are typically obtained after 1 min irradiation, see Figure 3 where a checker-board pattern is shown. The obtained patterns emphasize the potential application in terms of image photo-inscription, although both the response time and image sharpness leave room for optimization. These results are consistent with previously reported observations, ${ }^{[17]}$ though the required intensity is here few tens times smaller.

Importantly in terms of applicability, the patterning medium constituted by the photo-responsive material proved to be robust as it did not show significant sign of ageing after a few months, nor after many patterning/erasing cycles had been performed on the same sample. In addition, we stress that the all-optically driven processes occurring in our system are reversible and that the kinetics of return to equilibrium are fully determined by the intrinsic properties of the photo-responsive chiral dopant. This means that the write-and-erase features of our opto-molecular addressing of liquid crystals can be tuned and optimized by appropriate engineering of the molecular switch. ${ }^{[34]}$ In particular, the use of photoactive chiral molecular switches with thermal stability is expected to overcome present limitations in terms of perennial recording of localized chiral topological states. ${ }^{[35]}$
To conclude, we have demonstrated the subnanowatt optical generation, control and morphing of topological defect structures and textures in liquid crystalline materials. The proposed opto-molecular approach basically relies on the optical control of material chirality at the molecular scale, which triggers the supramolecular ordering. By achieving a drastic reduction of the required power budget with respect to state-of-the-art approaches, massively parallel optical writing of arbitrary localized microstructures can be envisioned using a light source with moderate power, for instance by using diffractive optical elements or lithographic exposure. Moreover, we showed that optical winding transition can be triggered over large areas, which holds potential for scaling up. Our results also suggest that previous experiments involving the generation of topological structures and optical phase singularities based on chiral anisotropic fluids can be fruitfully revisited with photoresponsive liquid crystals, thereby enriching the already welldeveloped stimuli-responsive materials toolbox. ${ }^{[36]}$

\section{Experimental Section}

All liquid crystal cells were purchased from E.H.C. Co., Ltd, Japan. The nematic liquid crystal mixtures E7 and MLC-2048, and the chiral dopant $\mathbf{S 8 1 1}$ were supplied by Merck. All chemical reagents used for the synthesis of the chiral dopants were purchased from Sigma-Aldrich. The ${ }^{1} \mathrm{H}$ NMR spectra were recorded on a $400 \mathrm{MHz}$ spectrometer in $\mathrm{CDCl}_{3}$, and they were in accordance with literature data.

The synthesis of the photo-responsive chiral dopant $\mathbf{m}$ was carried out following a procedure adapted from Ref. ${ }^{[27]}$ and ${ }^{[37]}$ for the upper-half. Subsequently, the two enantiomers of $\mathbf{m}$ were separated by chiral HPLC on a CHIRALPAK AD-H column using a methanol/ethanol (1:1) mobile phase, affording $>99 \%$ ee of enantiomer $\mathbf{m}$ with $(R, M)$-configuration and $>99 \%$ ee of enantiomer $m$ with $(S, P)$-configuration, in a 1:1 ratio. The helical twisting power (HTP) of $\mathbf{m}$ in $(S, P$-configuration) was measured in E7 using a Grandjean-Cano wedge cell and provided the following values: $\quad \operatorname{HTP}_{w t} \%(\mathrm{~m})=+66.04 \mu \mathrm{m}^{-1}, \quad \mathrm{HTP}_{\mathrm{wt}} \%\left(\mathrm{~m}^{*}\right)=-41.9 \mu \mathrm{m}^{-1}$. These results are in accordance with data reported by literature. ${ }^{[38]} \mathrm{H}$-NMR $(400 \mathrm{MHz}, \mathrm{CDCl} 3): \delta 3.15\left(\mathrm{~d},{ }^{2} \mathrm{~J}_{\mathrm{HH}}=16.0 \mathrm{~Hz}\right.$, $\left.1 \mathrm{H}, \mathrm{CH}_{2}\right), 3.91\left(\mathrm{dd},{ }^{2} \mathrm{~J}_{\mathrm{HH}}=16.0 \mathrm{~Hz},{ }^{3} \mathrm{~J}_{\mathrm{HH}}=8 \mathrm{~Hz}, 1 \mathrm{H}, \mathrm{CH}_{2}\right), 5.30$ $\left(\mathrm{d},{ }^{3} \mathrm{~J}_{\mathrm{HH}}=8 \mathrm{~Hz}, 1 \mathrm{H}, \mathrm{CH}\right), 6.72-6.80(\mathrm{~m}, 2 \mathrm{H}), 7.02-7.20(\mathrm{~m}, 7 \mathrm{H}), 7.24$ $\left(\mathrm{t},{ }^{3} J_{H H}=7 \mathrm{~Hz}, 1 \mathrm{H}\right), 7.33-7.45(\mathrm{~m}, 4 \mathrm{H}), 7.69\left(\mathrm{~d},{ }^{3} J_{H H}=8 \mathrm{~Hz}, 1 \mathrm{H}\right)$, $7.73\left(\mathrm{~d},{ }^{3} \mathrm{~J}_{\mathrm{HH}}=4 \mathrm{~Hz}, 1 \mathrm{H}\right), 7.80\left(\mathrm{~d}^{3}{ }^{3} \mathrm{~J}_{\mathrm{HH}}=8 \mathrm{~Hz}, 1 \mathrm{H}\right), 7.87\left(\mathrm{~d},{ }^{3} \mathrm{~J}_{\mathrm{HH}}=8\right.$ $\mathrm{Hz}, 1 \mathrm{H}), 7.93\left(\mathrm{~d}^{3}{ }^{3} \mathrm{~J}_{\mathrm{HH}}=8 \mathrm{~Hz}, 1 \mathrm{H}\right)$.

The synthesis of the shape-persistent chiral dopant $(R)-2,2^{\prime}-(1,3$ propylenedioxy)-1,1'-binaphthalene was carried out as described earlier in Ref. ${ }^{[39]}$. The HTP of (R)-2,2'-(1,3-propylenedioxy)-1,1'-binaphthalene was measured in E7 using a Grandjean-Cano wedge cell and provided the value $\mathrm{HTP}_{\mathrm{wt}} \%=-65.5 \mu \mathrm{m}^{-1}$. ${ }^{1} \mathrm{H}$ NMR $\left(400 \mathrm{MHz}, \mathrm{CDCl}_{3}\right): \delta 7.96(\mathrm{~d}$, $2 \mathrm{H}), 7.89(\mathrm{~d}, 2 \mathrm{H}), 7.47(\mathrm{~d}, 2 \mathrm{H}), 7.37(\mathrm{~m}, 2 \mathrm{H}), 7.22-7.28(\mathrm{~m}, 4 \mathrm{H}), 4.37$. $4.44(\mathrm{~m}, 4 \mathrm{H}), 1.94-1.99(\mathrm{~m}, 2 \mathrm{H})$.

\section{Acknowledgments}

We thank C. A. Hommersom for the synthesis of $(R)-2,2^{\prime}-(1,3-$ propylenedioxy)-1, $1^{\prime}$-binaphthalene. This work is financially supported by DGA contract 201160083 (C.L. and E.B.), by the Dutch Science Foundation NWO under a Vidi grant (S.I and N.K.) and by the European Research Council under the ERC Starting Grant 307784 (N.K). 
[1] E. Brasselet, N. Murazawa, H. Misawa, S. Juodkazis, Phys. Rev. Lett. 2009, 103, 103903.

[2] E. Brasselet, C. Loussert, Opt. Lett. 2011, 36, 719.

[3] E. Brasselet, Phys. Rev. Lett. 2012, 108, 087801.

[4] R. Barboza, U. Bortolozzo, G. Assanto, E. Vidal-Henriquez, M. G. Clerc, S. Residori, Phys. Rev. Lett. 2012, 109, 143901.

[5] C. Loussert, U. Delabre, E. Brasselet, Phys. Rev. Lett. 2013, 111, 037802.

[6] I. I. Smalyukh, Y. Lansac, N. A. Clark, R. P. Trivedi, Nature Mater. 2010, 9, 139

[7] C. Loussert, E. Brasselet, Appl. Phys. Lett. 2014, 104, 051911.

[8] W. E. L. Haas, J. E. Adams, Appl. Phys. Lett. 1974, 25, 263.

[9] M. Kawachi, O. Kogure, Y. Kato, Jpn. J. Appl. Phys. 1974, 13, 1457.

[10] P. Oswald, J. Baudry, S. Pirkl, Phys. Rep. 2000, 337, 67.

[11] I. I. Smalyukh, B. I. Senyuk, P. Palffy-Muhoray, O. D. Lavrentovich, H. Huang, E. C. Gartland,V. H. Bodnar, T. Kosa, B. Taheri, Phys. Rev. E 2005, 72, 061707.

[12] I. I. Smalyukh, D. Kaputa, A. V. Kachynski, A. N. Kuzmin, P. J. Ackerman, C. W. Twombly, T. Lee, R. P. Trivedi, P. N. Prasad, Opt. Express 2012, 20, 6870.

[13] J. S. Evans, P. J. Ackerman, D. J. Broer, J. van de Lagemaat, I. I. Smalyukh, Phys. Rev. E 2013, 87, 032503.

[14] P. J. Ackerman, Z. Qi, Y. Lin, C. W. Twombly, M. J. Laviada, Y. Lansac, I. I. Smalyukh, Sci. Rep. 2012, 2, 414.

[15] B. Yang, E. Brasselet, J. Opt. 2013, 15, 044021.

[16] O. Trushkevych, P. Ackerman, W. A. Crossland, I. I. Smalyukh, Appl. Phys. Lett. 2010, 97, 201906.

[17] I. Grozdovskyy, O. Yaroshchuk, M. Serbina, R. Yamaguchi, Opt. Express 2012, 20, 3499.

[18] Q. Li, Y. Li, J. Ma, D.-K. Yang, T. J. White, T. J. Bunning, Adv. Mater. 2011, 23, 5069.

[19] B. L. Feringa, N. P. M. Huck, A. M. Schoevaars, Adv. Mater. 1996, $8,681$.

[20] B. L. Feringa, W. R. Browne, Molecular Switches, Wiley-VCH, Weinheim, Germany 2011.
[21] T. J. White, S. A. Cazzell, A. S. Freer, D.-K. Yang, L. Sukhomlinova, L. Su, T. Kosa, B. Taheri, T. J. Bunning, Adv. Mater. 2011, 23, 1389.

[22] C. L. van Oosten, C. W. M. Bastiaansen, D. J. Broer, Nat. Mater. $2009,8,677$.

[23] S. Iamsaard, S. J. Aßhoff, B. Matt, T. Kudernac, J. J. L. M. Cornelissen, S. P. Fletcher, N. Katsonis, Nature Chem. 2014, 6, 229.

[24] D. J. Broer, C. M. W. Bastiaansen, M. G. Debije, A. P. H. J. Schenning, Angew. Chem. Int. Ed. 2012, 51, 7102.

[25] R. D. Kamien, J. V. Selinger, J. Phys.: Condens. Matter 2001, 13, R1.

[26] B. Y. Zel'dovich, N. V. Tabiryan, JETP Lett. 1981, 34, 406.

[27] J. Vicario, M. Walko, A. Meetsma, B. L. Feringa, J. Am. Chem. Soc. 2006, 128, 5127

[28] R. Eelkema, M. M. Pollard, J. Vicario, N. Katsonis, B. S. Ramon, C. W. M. Bastiaansen, D. J. Broer, B. L. Feringa, Nature 2006, 440, 163.

[29] Y. Wang, Q. Li, Adv. Mater. 2012, 24, 1926.

[30] P. Oswald, P. Pieranski, Nematic and Cholesteric Liquid Crystals: Concepts and Physical Properties Illustrated by Experiments, Taylor \& Francis, Boca Raton, FL, 2005.

[31] B. G. Chen, P. J. Ackerman, G. P. Alexander, R. D. Kamien, I. I. Smalyukh, Phys. Rev. Lett. 2013, 110, 237801.

[32] J.-S. Hsu, B.-J. Liang, S.-H. Chen, Appl. Phys. Lett. 2006, 89, 051920.

[33] P. G. de Gennes, J. Prost, The Physics of Liquid Crystals, Clarendon Press, 1995, 2nd ed.

[34] S. J. Aßhoff, S. lamsaard, A. Bosco, J. J. L. M. Cornelissen, B. L. Feringa, N. Katsonis, Chem. Commun. 2013, 49, 4256.

[35] Y. Li, C. Xue, M. Wang, A. Urbas, Q. Li, Angew. Chem. Int. Ed. 2013, 52, 13703.

[36] Q. Li, ed., Intelligent Stimuli-Responsive Materials: From Well-Defined Nanostructures to Applications, John Wiley \& Sons, Hoboken, New Jersey, 2012.

[37] A. S. Lubbe, N. Ruangsupapichat, G. Caroli, B. L. Feringa, J. Org. Chem. 2011, 76, 8599.

[38] R. Eelkema, B. L. Feringa, Org. Biomol. Chem. 2006, 4, 3729.

[39] J. J. G. S. van Es, H. A. M. Biemans, E. W. Meijer, Tetrahedron: Asymm. 1997, 8, 1825. 